\title{
Sequence Analysis of Putative potB, potC, and potD Genes from Serratia rubidae
}

\author{
SONY SUHANDONO*, RASI FITRIA, AND ERNAWATI ARIFIN GIRI RACHMAN \\ School of Life Sciences and Technology, \\ Institut Teknologi Bandung, Jalan Ganesa 10, Bandung 40132, Indonesia
}

\begin{abstract}
Amplification of putative potBCD genes from Serratia rubidae was conducted by PCR using a pair of primers FERC and RERC. A fragment with $\sim 1800 \mathrm{bp}$ size was ligated to pGEM-T Easy vector then cloned to competent Escherichia coli DH5 $\alpha$. The recombinant plasmid was sequenced using SP6, T7 and two internal primers, FPC and RPC. A sequence similarity search and analysis was performed with the BLASTN program. The sequence was found to have $83 \%$ similarity to pot $A B C D$ genes from $E$. coli. Those genes encoded a spermidinepreferential uptake system that consists of four kinds of protein: PotA is a membrane-associated ATPase, PotB and PotC are transmembrane proteins that form channels, and PotD is a periplasmic substrate-binding protein. Alignment analysis showed that the isolated clone consisted of $\operatorname{pot} B$ (partial), $\operatorname{pot} C$ (full length) and potD (partial). The sequences for potBCD genes from $S$. rubidae are not available in the NCBI database. Furthermore, we have submitted this sequence, potBCD from S. rubidae, on GeneBank with Acc. number FJ447342.
\end{abstract}

Key words: Serratia rubidae, spermidine-preferential uptake system, PotABCD, polyamine

Serratia is a prominent opportunistic pathogen (Holt et al. 1994) that infects plants and animals, including human. The bacteria belongs to Gram negative bacteria group (Holt et al. 1994). Ten species are presently known to belong in the genus Serratia. These species are $S$. marcescens, $S$. liquefaciens, S. proteamaculans, S. grimesii, S. plymuthica, S. rubidae, S. odorifera, S. ficaria, S. entomophila, and $S$. fonticola (Grimont and Grimont 2006).

Serratia rubidae has been isolated from coconuts, vegetables and fresh cheese as a dairy product (Malcata 1998). In the previous research, S. rubidae was found in human clinical specimens such as blood, bile (Ewing et al. 1973), urine (Menezes et al. 2004), respiratory tract (sputum) (Johnson and Ellner 1974), and feces (Farmer et al. 1985).

Serratia rubidae may be responsible for nosocomial infection in particular sepsis (Stock et al. 2003), colangitis, septicemia and urine infection (Menezes et al. 2004). It was shown to be an invasive pathogen (Ursua et al. 1996). Hospital infection due to $S$. rubidae is regarded to be associated with the consumption of contaminated coconuts or vegetable salads (Stock et al. 2003) and the bacteria may also be carried by hospital gowns (Pilonetto et al. 2004). Serratia produces multiple enterotoxic factors that may be significant in the understanding of its pathology (Singh et al. 1996). In addition, pathogenicity factors found in Serratia strains are the formation of fimbriae, the production of potent siderophores, the presence of cell wall antigens, the ability to resist the bactericidal action of serum and the production of proteases (Grimont and Grimont 2006).

In the recent studies, it was reported that some pathogenic bacteria had genes with homology to a polyamine transporter (Pot) operon in Escherichia coli. Proteins that are encoded by those genes have been implicated in the pathogenesis of bacteria, such as pneumococcal infection (Ware et al. 2006). When a

\footnotetext{
*Corresponding author, Phone/Fax: +62-22-2511575; Email: sony@sith.itb.ac.id
}

pathogen invades host cells, it must adapt quickly to a new environment to multiply and evade the host immune system. Polyamines would be actively involved in those cellular processes. Polyamines (spermidine, spermine, putrescine and cadaverine) are small polycationic compounds present in all living organisms (Tabor and Tabor 1985). They are essential for normal cell growth due to their role in cell proliferation and differentiation (Igarashi and Kashiwagi 1999). The polyamine content in cells is maintained by biosynthesis, degradation and transport from the environment (Igarashi and Kashiwagi 1999).

In $E$. coli, the genes for three different polyamine transport systems have been cloned and characterized (Kashiwagi et al. 1990). Two uptake systems (spermidinepreferential or PotABCD and putrescine-specific or PotFGHI) were ABC (ATP binding cassette) transporters (Kashiwagi et al. 2002). The third transport system, catalyzed by PotE, mediates both the uptake and the excretion of putrescine (Kashiwagi et al. 1992). Although polyamine transport systems may play an important role in pathogenesis and immunity of pathogenic bacteria, polyamine uptake in $S$. rubidae has not been well studied. In this study, we isolated three genes on pot $A B C D$ operon from $S$. rubidae, namely pot $B$, pot $C$ and potD.

Spermidine-preferential uptake systems specifically bind either spermidine or putrescine, with a higher affinity for spermidine (Kashiwagi et al. 1996). This polyamine transport system consists of four kinds of protein: PotA, PotB, PotC and PotD (Kashiwagi et al. 1990). PotA is a membrane-associated ATPase, PotB and PotC are integral membrane proteins that form a polyamine-specific transport channel and PotD is a surface-associated, polyaminebinding protein (Ware et al. 2006). The calculated molecular weights for these proteins were 43 026, 31060,29109 and 38865 Da, respectively (Furuchi et al. 1991). The PotD affinity for putrescine is ten-fold lower than spermidine (Igarashi and Kashiwagi 1999). Immunization with recombinant PotD on spermidine-preferential uptake system was proven to induce a vigorous antibody response in mice 
against pneumococcal infection (Shah and Swiatlo 2006). The purpose of this research is to isolate the pot $A B C D$ operon from $S$. rubidae.

\section{MATERIALS AND METHODS}

Bacterial Strain. The strain used in this research was isolated from degraded vegetables and identified by The Microbiology Laboratory, School of Life Sciences and Technology, Institut Teknologi Bandung.

Genomic and Gene Isolation. Chromosomal DNA from $S$. rubidae was isolated from cells harvested at their early stationary growth phase. The isolation protocol follows that of Bronke et al. (2001). The potABCD operon was amplified by PCR with genomic DNA from $S$. rubidae serving as a template. A pair of primers FERC (5'ATGGTGGAAGCAGGCTGTTT-3') and RERC (5'ATAAATGCCCGACTGCCA-3') were used in this reaction. The reaction included 25 cycles with denaturation temperature of $95^{\circ} \mathrm{C}$, annealing temperature of $49^{\circ} \mathrm{C}$ and elongation temperature of $72^{\circ} \mathrm{C}$. PCR product with $\sim 1800$ bp size was purified using Geneaid Gel/PCR-DNAFragment-Extraction Kit.

Cloning and Transformation. The purified PCR product was ligated to pGEM-T Easy vector (Promega) using the standard protocol. Furthermore, the vector was transformed into competent $E$. coli $\mathrm{DH} 5 \alpha$ cells with heat shock method (Sambrook et al. 1989). The transformans were plated onto LB medium that contained ampicillin, IPTG and X-Gal and then incubated overnight at $37^{\circ} \mathrm{C}$.

Plasmid Isolation. A single white colony (putative clone with DNA insert) was picked and inoculated to LB medium supplemented with $100 \mu \mathrm{g} \mathrm{mL}^{-1}$ ampicillin as a selection antibiotic. The bacterial culture was incubated overnight (16 hours) at $37^{\circ} \mathrm{C}$ with shaking at $250 \mathrm{rpm}$. Plasmid isolation was performed using GeneJET Plasmid Miniprep Kit (Fermentas) as described in the product manual. The existence of insert DNA was confirmed by cutting the plasmid with EcoRI as it was one of the restriction enzyme that cut the pGEM-T Easy vector at the multiple cloning sites.

Sequencing. The DNA plasmid containing the insert was sequenced by Macrogen, Inc. Korea using SP6 primer, T7 primer, and two internal primers, FPC and RPC. Nucleic acid and deduced asam amino sequences were analyzed by BiOEDIT and ClustalW programs. Domain prediction of putatif PotB protein was performed by ScanProsite, online pogram from www.expasay.ch/tools.

\section{RESULTS}

The genomic DNA samples of $S$. rubidae, as a template in the PCR reaction, were successfully extracted. Their concentration ranged from 2-6.8 $\mu \mathrm{g} \mu \mathrm{L}^{-1}$, and the DNAprotein ratios were around 1.75-1.79. Fig 1 (left) showed some DNA fragments amplified by the PCR method as given in the Materials and Methods.

The purified fragment (size $\sim 1800 \mathrm{bp}$ ) was ligated to pGEM-T Easy as a cloning vector. Blue-white colony
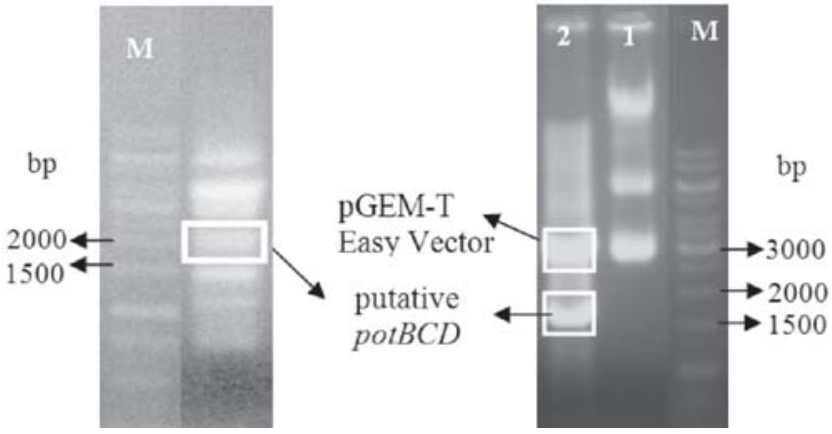

Fig 1 (Left) PCR reaction products. Putative pot $B C D$ were sized approximately 1800 bp; (Right) Line 1 : Recombinant plasmid uncut. Line 2 : Recombinant plasmid cut by Eco RI. M: marker (1 kb DNA ladder).

screening was performed by employing IPTG and X-Gal within the LB-medium. We also used ampicillin as selective antibiotic for the E. coli transformant. A single white colony, which is the putative clone with the DNA insert, was then inoculated into fresh LB-medium for the next stage. Confirmation of DNA insertion was conducted by cutting the plasmid using EcoRI. Line 2 at the electrophoregram (Fig 1, right) showed 2 fragments of DNA, the upper fragment was pGEM-T Easy vector, while the fragment with smaller molecular mass (lower fragment) was the DNA insert. Sequencing using Automatic DNA sequencer showed that the DNA insert was a 1868 bp (Fig 2).

\section{DISCUSSION}

Sequence analysis revealed that length of the fragment was 1868 bp (Fig 2). The potB, potC and potD was also found in E.coli (Blattner et al.1997; Kashiwagi et al. 2002). The sequence had $83 \%$ similarity to pot $A$, pot $B$, pot $C$ and pot $D$ genes from $E$. coli (Acc. number M64519.1) with expectation value 0 and $1 \%(34 / 1870)$ gaps. Alignment analysis showed that the isolated clone consisted of potB (partial), potC (full length) and potD (partial). The $\operatorname{pot} B, \operatorname{pot} C$, and potD genes were located at 14-594, 5901382 and $1378-1860$ bp on the nucleotide sequence, respectively. The open reading frames for those genes partially overlapped.

We obtained the amino acid sequence of these genes using in-silico translation of the nucleotide sequence by BioEdit software. The amino acid sequence analysis was performed by BLASTP program. Putative PotB from Serratia rubidae had $95 \%$ similarity to spermidine/ putrescine $\mathrm{ABC}$ transporter membrane protein (PotB) from Citrobacteri koseri ATCC BAA-895 (Acc. number ABV13055.1). Putative PotC had 96\% similarity to spermidine/putrescine $\mathrm{ABC}$ transporter membrane protein (PotC) Shigella dysenteriae Sd197 (Acc. number ABB 62123.1). In addition, putative PotD had $89 \%$ similarity to spermidine/putrescine $\mathrm{ABC}$ transporter periplasmic substrate-binding protein (PotD) from Klebsiella pneumoniae subsp. pneumoniae MGH (Acc. number ABR 76562.1).

PotB and PotC are the membrane components of the ABC transporter (Igarashi 1999). Domain prediction of putatif PotB protein was performed by ScanProsite, an 


\section{$R E R C$}

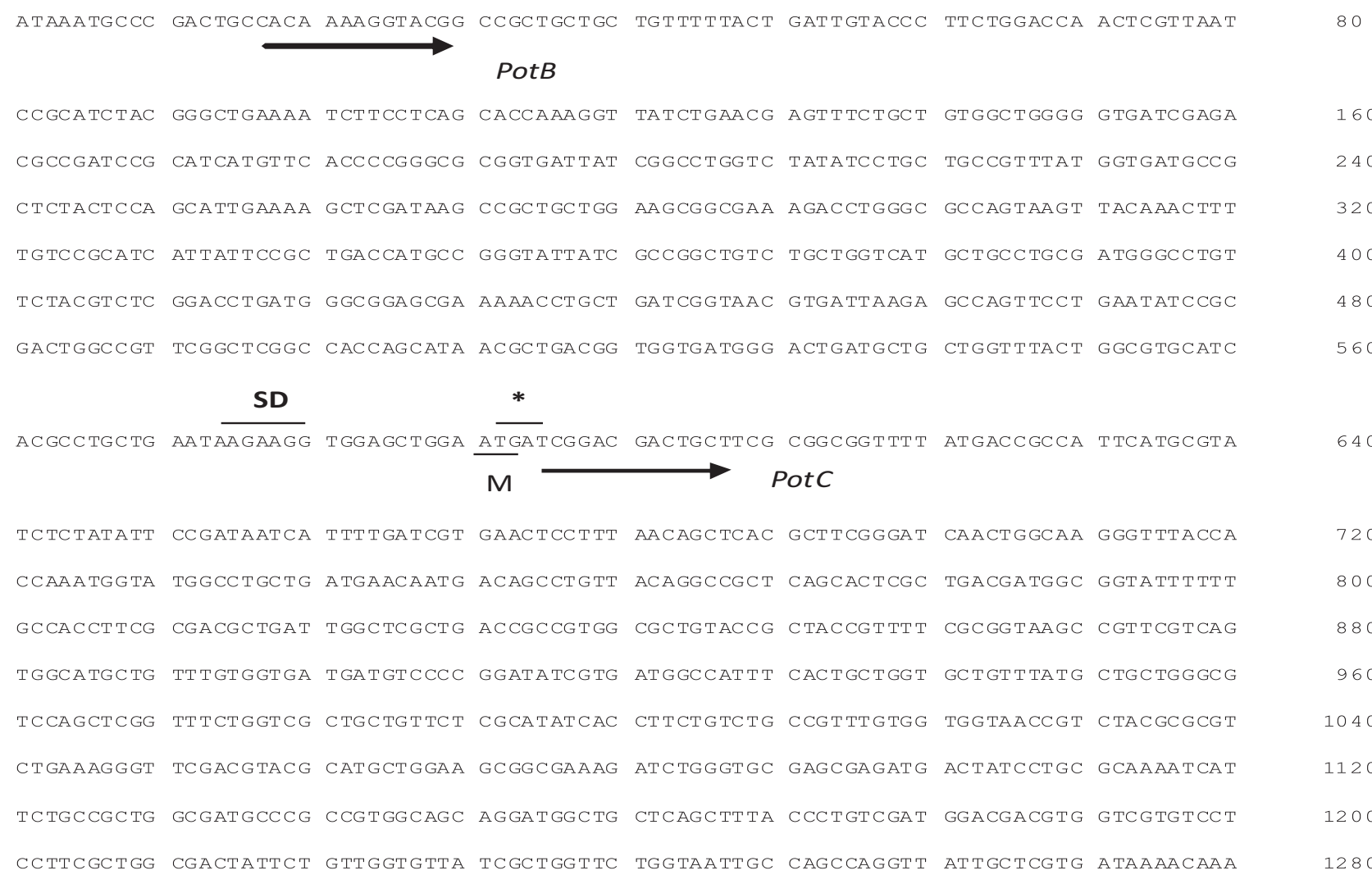

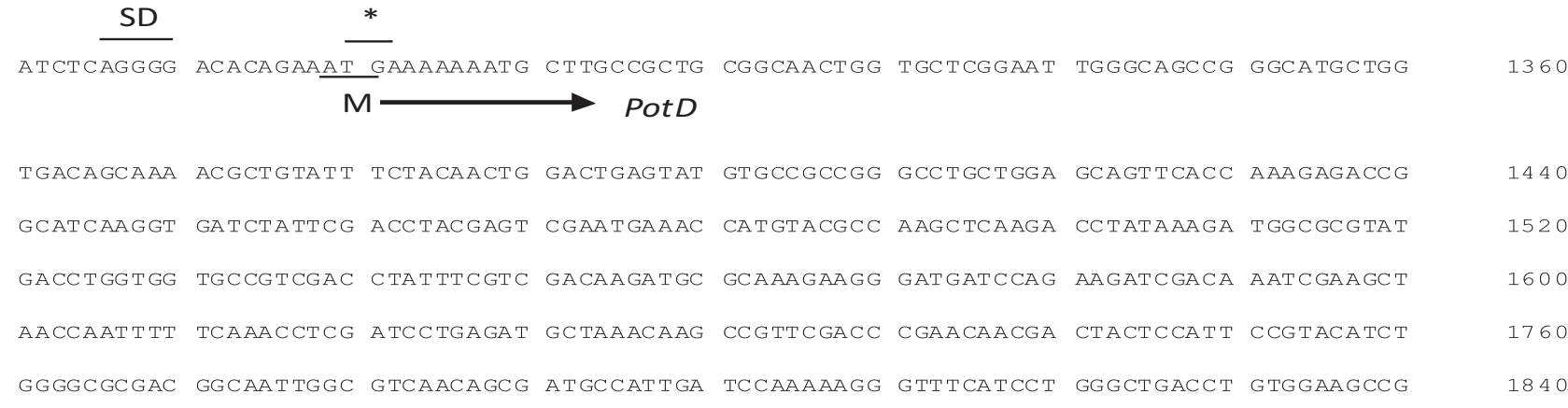

GAATACAAAA ACAGCCTGCT TCCACCAT1 868

FERC

Fig 2 Nucleotide sequence of potB, potC and potD genes from $S$. rubidae. SD denotes putative Shine- Delgarno sequence. Codon start indicated by "M" and codon stop indicated by "*”. Grey boxes indicate a pair of primers.

online program available at www.expasay.ch/tools. It was ascertained that the mentioned protein belonged to the $\mathrm{ABC}$ transporter-integral-membrane type-1 domain. In addition, putative PotC protein was analyzed by ScanProsite and InterProScan from EMBL. Putative PotC from $S$. rubidae belonged to the ABC transporter integral membrane type-1 domain. In E. coli, both proteins (PotB and PotC) are membrane proteins that form a transport channel on the spermidine-preferential uptake system. Protein domain prediction for PotD could not be undertaken because the sequence obtained was not adequate to be analyzed with previous program.

The amino acid sequence of each of the genes was aligned with the amino acid sequence of PotB, PotC and
PotD from E. coli using ClustalW. As mentioned above, in E. coli PotB and PotC are membrane proteins. The prediction of six transmembrane segments was in line with the finding of Furuchi et al. (1991). The alignment analysis results (Fig 3) indicate that fragments of putative PotB from S. rubidae has only four transmembrane segments (TM 3TM 6). It had $95 \%$ similarity to PotB protein from $E$. coli. It appears that the putative potB was not the complete isolate, only its carboxyl terminus.

Fig 4 shows the alignment analysis between putative PotC from $S$. rubidae and PotC from E. coli. Both sequences had $94 \%$ similarity according to the alignment score. The putative PotC from $S$. rubidae lacks one amino acid compared with its PotC counterpart from $E$. coli. 
TM1
PotB_S.rubidae

PotB_E.coli

PotB_S.rubidae

PotB E.coli

PotB_S.rubidae

PotB E.coli

PotB_S.rubidae

PotB_E.coli
PotB_S.rubidae

PotB_E.coli :MIVTIVGWLVLFVFLPNLMIIGTSFLTRDDASFVKMVFTLDNYTRLLDPLYFEVLLHSLN
TM2 TM3

PQKVRPLLLFLLIVPFWTNSLIRIYGLKIFLSTKGYL 37

:MALIATLACLVLGYPFAWFL AKLPHKVRPLLLFLLIVPFWTNSLIRIYGLKIFLSTKGYL 120

TM4

:NEFLLWLGVIETPIRIMFTP GAVIIGLVYILLPF MVMPLY SSIEKLDK PLLEAAKDLGAS 97 :NEFLLWLGVIDTPIRIMFTP SAVIIGLVYILLPF MVMPLY SSIEKLDKPLLEAARDLGAS 180

\section{TM5}

:KLQTFVRIIIPLT MPGIIAGCLLVMLPA MGLFYVSDLMGGAKNLLIGNVIKSQFLNIRDW 157 :KLQTFI RIIIPLTMPGIIAGCLLVMLPA MGLFYVSDLMGGAKNLLIGNVIKVQFLNIRDW 240

TM6

:PFGSATSITLTVVMGLMLLVY WRASRLLNKKVELE 192

:PFGAATSITLTI VMGLMLLVY WRASRLLNKKVELE 275

Fig 3 Sequence alignment of putative PotB protein from S. rubidae and PotB from $E$. coli Acc Number M 64519.1 which were aligned using the Clustal W algorithm. Grey boxes indicate the six predicted transmembrane segments (TM1 to TM6). Consensus symbols; identical residues: "*", conserved substitutions: ":", semi-conserved substitutions:".".

\section{TM1}

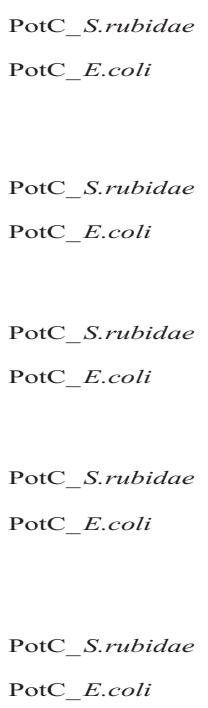

Fig 4 Sequence alignment of putative PotC protein from S. rubidae and PotC from E. coli Acc number M64519.1 using the Clustal W algorithm. Grey boxes indicate the six predicted transmembrane segments (TM 1 to TM 6). Consensus symbols; identical residues: "*", conserved substitutions: ":", semiconserved substitutions:".".

Alignment analysis revealed that fragments of putative PotD from $S$. rubidae had $86 \%$ similarity to PotD protein from E. coli. Fourteen residues of the PotD protein (Trp 34, Thr 35, Glu 36, Tyr 37, Ser 83, Tyr 85, Asp 168, Glu 171, Ser 211, Trp 229, Trp 255, Asp 257, Tyr 293 and Gln 327) in $E$. coli are involved in spermidine binding. Six of them (Trp 34, Thr 35, Glu 36, Tyr 37, Ser 83 and Tyr 85) were found in the putative PotD from $S$. rubidae (shown in grey boxes, Fig 5). It is predicted that the isolated putative pot $D$ gene represents only its amino terminus domain. The PotD protein and the periplasmic proteins consist of repeated $\beta-\alpha-\beta$ units. The isolated putative $\operatorname{pot} D$ had five $\beta$ units and three $\alpha$ units (Fig 5).

In $E$. coli, the potA gene is located in at the upstream region of $\operatorname{pot} A B C D$ operon, followed by $\operatorname{pot} B, \operatorname{pot} C$ and potD genes. The size of pot $A B C D$ operon from E. coli is approximately 4300 bp (Furuchi et al. 1991), thus gene walking experiment should be conducted in the future to identify the full length of pot $A B C D$ operon from S. rubidae.

We conclude that the isolated potBCD genes from $S$. rubidae consisted of partial potB, full length pot $C$ and 
PotD S.rubidae PotD_E.coli

PotD S.rubidae PotD_E.coli

PotD_S.rubidae PotD_E.coli

PotD S.rubidae PotD_E.coli

PotD S.rubidae PotD_E.coli

PotD S.rubidae PotD_E.coli
:MKKM - - LAAAATGARNWAAAHAGDCKTLYFYN WTEYVPPGLLEQFTKETGIKVIYSTYE 57 :MKKWSRHLLAAGALA LGMSAAHADDNNTLYFY N WTEYVPPGLLEQFTKETGIKVIYSTYE 60

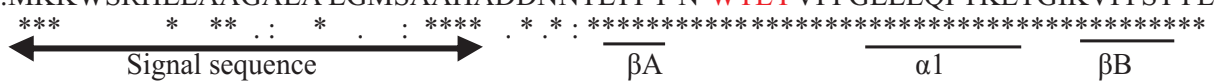

:SNETMYAKLKTYKDGAYDLVVP STYF VDKMRKEGMIQKIDKSKLTNFSNLDPEMLNKPED :SNETMYAKLKTYKDGAYDLVVP STYYVDKMRKEGMIQKIDKSKLTNFSNLDPDMLNKPED $* * * * * * * * * * * * * * * * * * * * * * * * * * * * * * * * *: * * * * * * * * * * * * * * * * * * * * * * * * * * * * * * * * * * * . . * * * * * * * * *$ 117 $\frac{2}{\alpha \mathrm{B}} \frac{: \ldots 3}{\alpha 3}$

PNNDYSIPYIWGATAIGVNSDA I DPKRVSSWADLWKPEYKNSL 160 :PNNDYSIPYIWGATAIGVNGDAVDPKRVTSWADLWKPEYKGSLLLTD DAREVFQMALRKL 180 $\frac{* * * * * * * * * * * * * * * * * * * * * * * *}{\beta \mathrm{D}}$ $\cdot \frac{* *}{\beta F}$

$\alpha 4$

:GYSGNTTDPKEIEAAYNELKKLMPNVAAFNSDNPANPYMEGEVNLGMIWNGSAFVARQAG

$\frac{\text { TPIDVVWPKEGGIFWMDSLAIPANAKNKEGALKLINFLLRPDVAKQVAETIG YPTPNLAA }}{\beta \mathrm{I}} \frac{\frac{\text { YJ }}{\alpha 8}}{\alpha 10}$

Fig 5 Sequence alignment between putative PotD protein from S. rubidae and PotD from E. coli Acc. number M 64519.1 Based on Sugiyama et al. (1996) the active residue which binds to spermidine are indicated by grey boxes. Consensus symbols; identical residues: "*", conserved substitutions: ":", semiconserved substitutions:".".

partial potD. We have submitted this sequence, potBCD from $S$. rubidae, on GeneBank with Acc. number FJ447342.

\section{ACKNOWLEDGEMENT}

We thank Sri Harjati Suhardi from SITH ITB and Puspita Lisdiyanti from Puslit Bioteknologi LIPI for critical comments on the manuscript. We also appreciate Tati Kristianti and M Bahrelfi Belafiff for their helpful comments and advice. This study was funded by Indonesion agency for agricultural research and development.

\section{REFERENCES}

Blattner FR, Plunkett G III, Bloch CA, Perna NT, Burland V, Riley M, Collado-Vides J, Glasner JD, Rode CK, Mayhew GF, Gregor J, Davis NW, Kirkpatrick HA, Goeden MA, Rose DJ, Mau B, Shao Y. 1997. Science 277:1453-74.

Bronke F, Hajirezaei M, Sonnewald U. 2001. Cloning and characterization of the gene cluster for palatinose metabolism from the phytopathogenic bacterium Erwinia rhapontici. J Bacteriol 183:2425-30.

Ewing WH, Davis BR, Fife MA, Lessel EF. 1973. Biochemical characterization of Serratia liquefaciens (Grimes and Hennerty) Bascomb et al. (Formerly Serratia and Enterobacter liquefaciens) and rubidaea (Stapp) comb. nov. designation of type and neotype strains. Int J Sys Bacteriol 23:217-25.

Farmer III BB, Davis BR, Hickman-Brenner FW, Mc Whorter A, Huntleycarter GP, Asbury MA, Riddle C, Wathen-Grady HG, Elias C, Fanning GR, Steigerwalt AG, O'hara CM, Morris GK, Smith PB, Brenner DJ. 1985. Biochemical identification of new species and biogroups of Enterobacteriaceae isolated from clinical specimens. J Clin Microbiol 21:46-76.

Furuchi T, Kashiwagi K, Kobayashi H, Igarashi K. 1991. Characteristics of the gene for a spermidine and putrescine transport system that maps at 15 min on the Escherichia coli Chromosome. J Biol Chem 266:2092833.
Grimont F, Grimont PAD. 2006. The genus Serratia. Prokaryotes 6:21944.

Holt GH, Krieg NR, Sneath PHA, Staley JT, Williams ST. 1994. Bergey's Manual of Determinative Bacteriology, $9^{\text {th }}$ ed. Baltimore: Williams \& Wilkins. p 175-287.

Igarashi K, Kashiwagi K. 1999. Polyamine transport in bacteria and yeast. Biochem J 344:633-42.

Johnson E, Ellner PD. 1974. Distribution of Serratia species in clinical specimens. Appl Microbiol 28:513-4.

Kashiwagi K, Hosokawa N, Furuchi T, Kobayashi H, Sasakawa C, Yoshikawa M, Igarashi K. 1990. Isolation of polyamine transportdeficient mutants of Escherichia coli and cloning of the genes for polyamine transport proteins. J Biol Chem 265:20893-7.

Kashiwagi K, Innami A, Zenda R, Tomitori H, Igarashi K. 2002. The ATPase activity and the functional domain of PotA, a component of the spermidine-preferential uptake system in Escherichia coli. J Biol Chem 277:24212-9.

Kashiwagi K, Miyamoto S, Suzuki F, Furuchi T, Kobayashi H, Igarashi K. 1992. Excretion of putrescine by the putrescine ornithine antiporter encoded by the potE gene of Escherichia coli. PNAS USA 89:4529-33.

Kashiwagi K, Pistocchi R, Shibuya S, Sugiyama S, Morikawa K, Igarashi K. 1996. Spermidine-preferential uptake system in Escherichia coli, identification of amino acids involved in polyamine binding in PotD protein. JBiol Chem 271:12205-8.

Malcata FX. 1998. Critical issues affecting the future of dairy industry, individual contributions in the scope of a global approach. J Dairy Sci 82:1595-611.

Menezes EA, Cezafar FC, Andrade MSS, Rocha MVAP, Cunha FA. 2004. Frequency of Serratia sp. in urine infections of intern patients in the Santa Casa de Misericórdia in Fortaleza. Revista Socieda Brasileira Med Trop 37:70-71.

Pilonetto M, Edvaldo, Rosa EAR, Brofman PRS, Baggio D, Calvário F, Schelp C, Nascimento A, Messias-Reason I. 2004. Hospital gowns as a vehicle for bacterial dissemination in an intensive care unit. Braz $\mathrm{J}$ Infect Dis 8:206-10.

Sambrook J, Fristch EF, Maniatis T. 1989. Molecular cloning: a laboratory manual. New Jersey: Cold Spring Harbor Laboratory Pr. 
Shah P, Swiatlo E. 2006. Immunization with polyamine transport protein PotD protects mice against systemic infection with Streptococcus pneumoniae. Infect Imun 74:5888-92.

Singh BR, Singh Y, Tiwari AK. 1996. Characterization of virulence factors of Serratia strains isolated from foods. Int J Food Microbiol 34:25966

Stock I, Burak S, Sherwood KJ, Grüger T, Wiedemann B. 2003. Natural antimicrobial susceptibilities of strains of 'unusual' Serratia species: $S$. ficaria, S. fonticola, S. odorifera, S. plymuthica and S. rubidaea. J Antimicrob Chemother 51:865-85.
Sugiyama S, Matsuo Y, Maenaka K, Vassylyev, Matsushima M, Kashiwagi K, Igarashi K, Morikawa K. 1996. The 1.8-A X-ray structure of the Escherichia coli PotD protein complexed with spermidine and the mechanism of polyamine binding. Prot Sci 5:1984-90.

Tabor CW, Tabor H. 1985. Polyamines in microorganisms. Microbiol Rev 49:81-99.

Ursua PR, Unzaga MJ, Melero P, Iturburu I, Ezpeleta C, Cisterna R. 1996. Serratia rubidae as an invasive pathogen. J Clin Microbiol 34:216-7.

Ware D, Jiang Y, Lin W, Swiatlo E. 2006. Involvement of potD in Streptococcus pneumoniae polyamine transport and pathogenesis. Infect Imun 74:352-61. 